\title{
Uso de Curvas de Transferência de Informação Extrínseca na Análise de Receptores Iterativos
}

\author{
Rafael de S. Marinho ${ }^{1}$, Jaime Portugheis ${ }^{2}$ e Daniel C. Cunha ${ }^{3}$
}

\begin{abstract}
Resumo-As curvas EXIT (do inglês, EXtrinsic Information Transfer Functions) demonstraram nos últimos anos sua capacidade para prever o comportamento da convergência da decodificação iterativa em diferentes sistemas de comunicação. Neste artigo utilizamos as funções EXIT para analisar um sistema de transmissão digital composto pela concatenação serial de um codificador LDPC, um modulador $M$-APSK e um codificador diferencial. O canal considerado é o AWGN (do inglês, Additive White Gaussian Noise) não-coerente de bloco. Da análise concluímos que códigos LDPC regulares possuem um desempenho relativamente próximo da capacidade de canal para um intervalo de coerência de canal suficientemente grande.
\end{abstract}

Palavras-Chave-Códigos LDPC, receptores iterativos, curvas EXIT.

Abstract-In the last years, EXtrinsic Information Transfer (EXIT) Charts have demonstrated their ability to predict convergence behavior of iterative decoding in several communications systems. In this paper, EXIT charts are used to analyze a digital transmission system consisting of a serial concatenation of LDPC encoder, $M$-APSK modulator and a differential encoder. A blockwise non-coherent AWGN channel is considered. From the analysis it is concluded that regular LDPC codes have performance relatively near capacity for sufficient large channel coherence interval.

Keywords - LDPC codes, iterative receivers, EXIT charts.

\section{INTRODUÇÃO}

As funções EXIT (do inglês, EXtrinsic Information Transfer), ou curvas EXIT, são uma ferramenta recente utilizada para analisar a decodificação iterativa com base na transferência de informação extrínseca entre os decodificadores componentes. Através delas pode-se prever a convergência da decodificação sem a necessidade de simulação computacional da probabilidade de erro. Tais curvas foram primeiramente definidas por S. ten Brink em [1] e, posteriormente, estendidas para códigos paralelamente concatenados (PCC, do inglês Parallel Concatenated Codes) [2]. Diante da boa previsão obtida para códigos PCC, a utilização dessas curvas passou a ser também considerada no projeto de códigos LDPC (do inglês, Low-Density Parity-Check Codes) [3] e de muitos outros processamentos iterativos existentes em sistemas de comunicação [4].

Neste artigo utilizamos as funções EXIT para analisar um sistema de transmissão digital composto pela concatenação

1 Fundação Paraense de Radiodifusão, Belém-PA, Brasil.

2 Departamento de Comunicações, FEEC, Universidade Estadual de Campinas (UNICAMP), Campinas-SP, Brasil.

3 Núcleo de Pesquisa em Telecomunicações, POLI, Universidade de Pernambuco (UPE), Recife-PE, Brasil.

E-mails: rsmarinho@gmail.com, dccunha@upe.poli.br. serial de um codificador LDPC, um modulador $M$-APSK e um codificador diferencial. O canal considerado é o AWGN não-coerente de bloco, um canal com memória limitada. Resultados para a probabilidade de erro de bit deste sistema foram apresentadas em [5] para a códigos LDPC regulares de comprimentos pequenos. A previsão da capacidade deste canal foi feita em [6]. O nosso intuito neste artigo é prever a capacidade da classe de códigos regulares e compará-la com os resultados de [5] e [6].

O artigo está organizado como se segue. A utilização de curvas EXIT para a análise de códigos LDPC é apresentada na Seção II. Na Seção III, a análise por meio de curvas EXIT é estendida para o receptor iterativo proposto. Na Seção IV, resultados numéricos são apresentados. Por fim, a Seção V é dedicada a conclusões e propostas de trabalhos futuros.

\section{CURVAS EXIT PARA CÓDIGOS LDPC}

Considere um código LDPC $C\left(w_{c}, w_{l}\right)$ de taxa $r_{c}=1-$ $w_{c} / w_{l}$, em que $w_{c}$ e $w_{l}$ são o peso das colunas e o peso das linhas da matriz de verificação de paridade do código, respectivamente. Este tipo de código pode ser representado por uma estrutura gráfica denominada grafo, que possui dois tipos de nós, quais sejam, os nós de variável (NV) e os nós de verificação de paridade (NP), ou simplesmente, nós de verificação. Os nós de variável representam os $n$ bits codificados de uma palavra-código, enquanto os nós de verificação representam as $(n-k)$ equações de verificação de paridade do código. Sendo assim, o grafo que representa o código LDPC pode ser visto como um decodificador iterativo, no qual os conjuntos de nós do grafo constituem os decodificadores componentes. Os conjuntos de nós de variável e de verificação são denominados Decodificador de Nós de Variável (DNV) e Decodificador de Nós de verificação de Paridade (DNP), respectivamente.

A decodificação iterativa é realizada por meio da passagem de mensagens entre o DNV e o DNP. A Fig.1 ilustra a estrutura do decodificador iterativo, na qual os blocos $\Pi$ e $\Pi^{-1}$ representam as conexões entre os nós de variável e de verificação, e $\widehat{\mathbf{x}}$, a palavra-código estimada na saída do decodificador. Os parâmetros $Z, E_{N V}, A_{N P}, A_{N V}$ e $E_{N P}$ representam as mensagens que são trocadas durante o procedimento de decodificação e são definidas como valores- $L$ ou logaritmos de razões de probabilidades (LLRs, do inglês Log-Likelihood Ratios). Enquanto o parâmetro $Z$ corresponde aos valores-L obtidos na saída do canal, os pares $\left(E_{N V}, A_{N P}\right)$ e $\left(E_{N P}, A_{N V}\right)$ correspondem, respectivamente, às mensagens trocadas entre os nós de variável e os nós de verificação e vice-versa. 


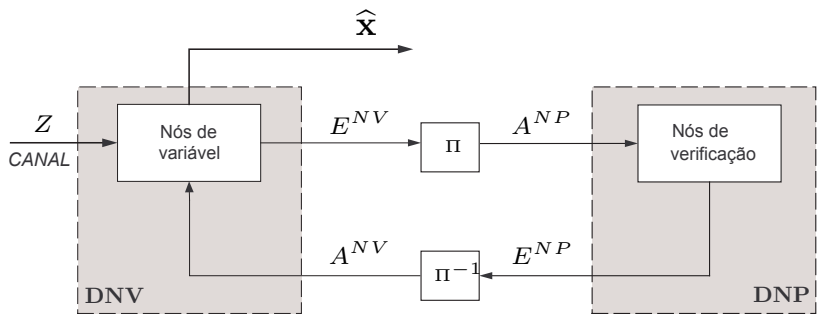

Fig. 1. Estrutura iterativa do decodificador LDPC.

Ao se analisar conjuntamente as atualizações das mensagens trocadas entre os decodificadores componentes (DNV e DNP), o procedimento de decodificação iterativa de um código LDPC pode ser interpretado como um sistema que utiliza dois tipos de informação com o objetivo de melhorar a estimativa da palavra-código transmitida. Tais informações são o conhecimento proveniente do canal (informação intrínseca) e o conhecimento obtido na iteração anterior da decodificação (informação extrínseca). A partir desses conhecimentos, o decodificador iterativo poderá realizar uma melhor estimação da palavra-código enviada, inclusive utilizando esta estimação como informação extrínseca para a próxima iteração. Uma das maneiras de se medir os conhecimentos anteriormente mencionados é utilizar a informação mútua entre uma Variável Aleatória (V.A.) $X$ que representa o bit transmitido e outra V.A. que representa uma mensagem associada ao mesmo bit [2]. A mensagem utilizada pode ser tanto do nó de variável para o nó de verificação $\left(E^{N V}, A^{N P}\right)$ quanto a recíproca $\left(E^{N P}, A^{N V}\right)$.

Seja $I_{E}=I(X ; E)$, a informação mútua calculada entre a V.A. $X$ e a V.A. $E$, que representa as mensagens de saída dos nós do grafo, ou seja, $I_{E}$ corresponde à informação extrínseca do sistema. Analogamente, considere que $I_{A}=I(X ; A)$ é a informação mútua entre $X$ e a V.A. $A$, que representa as mensagens de entrada dos nós. A informação mútua $I_{A}$ representa a informação a priori de um decodificador componente e corresponde à informação extrínseca do outro decodificador. Fixando-se os parâmetros do canal, podemos ver $I_{E}$ como função de $I_{A}$, i.e., $I_{E}=T\left(I_{A}\right)$. Esta função é conhecida como curva EXIT (do inglês, Extrinsic Information Transfer) e através dela podemos predizer o comportamento do decodificador.

\section{A. Curva EXIT do Decodificador de Nós de Variável}

Diz-se que um nó de variável possui grau $d_{v}$ caso exista $d_{v}$ nós de verificação conectados a ele. Desta forma, um nó de variável de grau $d_{v}$ recebe $\left(d_{v}+1\right)$ mensagens, pois existe uma mensagem adicional vinda do canal. Com base no algoritmo Soma-Produto (SP) [7], a mensagem de saída do $i$-ésimo nó de variável é obtida por meio da equação

$$
E_{i}^{N V}=Z_{i}+\sum_{j \neq i} A_{j}^{N V}
$$

em que $A_{j}^{N V}$ são as $d_{v}$ mensagens provenientes dos nós de verificação conectados ao $i$-ésimo nó de variável e $Z_{i}$ é a mensagem vinda do canal.

Assuma que a modulação BPSK (do inglês, Binary Phase Shift Keying) e o canal com ruído Gaussiano de variância $\sigma_{w}^{2}$ são considerados. Além disso, a relação sinal-ruído normalizada é definida como $E_{b} / N_{0}=1 /\left(2 r_{c} \sigma_{w}^{2}\right)$. Logo, a mensagem $Z_{i}$ é expressa como

$$
Z_{i}=\ln \left(\frac{p\left(y_{i} \mid x_{i}=+1\right)}{p\left(y_{i} \mid x_{i}=-1\right)}\right),
$$

em que $p\left(y_{i} \mid x_{i}\right)$ é a função densidade de probabilidade condicional da saída $y_{i}$ dada a entrada $x_{i}$.

Para o cálculo da função EXIT do DNV, as mensagens $Z \mathrm{e}$ $A^{N V}$ são consideradas V.A.s Gaussianas. Para as mensagens $Z$, a justificativa está na utilização do modelo do canal Gaussiano, enquanto que para as mensagens $A^{N V}$, a soma de várias mensagens, oriundas de valores- $L$, tipicamente possuem distribuições Gaussianas [2]. Assim, a informação mútua $I_{E}^{N V}$ é aproximada por

$I_{E}^{N V}\left(I_{A}^{N V}, d_{v}, \frac{E_{b}}{N_{0}}, r_{c}\right)=J\left(\sqrt{\left(d_{v}-1\right)\left[J^{-1}\left(I_{A}^{N V}\right)\right]^{2}+\sigma_{Z}^{2}}\right)$

em que $\sigma_{Z}^{2}$ é a variância de $Z$ condicionada a $X$, dada por

$$
\sigma_{Z}^{2}=\frac{4}{\sigma_{w}^{2}}=8 r_{c} \frac{E_{b}}{N_{0}} .
$$

A função $J(\cdot)$ corresponde à informação mútua entre a V.A. $X$ e os valores- $L$ na saída do canal, enquanto que $J^{-1}(\cdot)$ é a função inversa de $J(\cdot)$. Tais funções são definidas em [3].

\section{B. Curva EXIT do Decodificador de Nós de Verificação}

Assim como no caso dos nós de variável, um nó de verificação que possui conexão com $d_{p}$ nós de variável é dito um nó de grau $d_{p}$. Também baseada no algoritmo SP, a mensagem de saída do $j$-ésimo nó de verificação do DNP é dada pela expressão (vide [8])

$$
E_{j}^{N P}=\ln \left(\frac{1-\prod_{k \neq j} \frac{1-\exp \left(A_{k}^{N P}\right)}{1+\exp \left(A_{k}^{N P}\right)}}{1+\prod_{k \neq j} \frac{1-\exp \left(A_{k}^{N P}\right)}{1+\exp \left(A_{k}^{N P}\right)}}\right) .
$$

Mais uma vez, é possível aproximar a distribuição de probabilidades das mensagens na saída do DNP por uma distribuição Gaussiana, segundo [9] e [10]. Adicionalmente, existe uma propriedade de dualidade para o canal BEC (do inglês, Binary Erasure Channel), a qual determina que a curva EXIT $I_{E, P S}(\cdot)$ de um código de verificação de paridade simples, cujas palavras-código possuem comprimento $d_{p}$, em função da curva EXIT $I_{E, R E P}(\cdot)$ de um código de repetição de taxa $1 / d_{p}$ é dada por [4]

$$
I_{E, P S}\left(I_{A}, d_{p}\right)=1-I_{E, R E P}\left(1-I_{A}, d_{p}\right) .
$$

Por conveniência, a equação (6) será utilizada como uma aproximação para o cálculo da curva EXIT do DNP, conforme a expressão

$$
\begin{aligned}
I_{E}^{N P}\left(I_{A}^{N P}, d_{p}\right) & \approx 1-I_{E, R E P}\left(1-I_{A}^{N P}, d_{p}\right) \\
& =1-J\left(\sqrt{d_{p}-1} \cdot J^{-1}\left(1-I_{A}^{N P}\right)\right)(7)
\end{aligned}
$$

obtida fazendo-se $\sigma_{Z}^{2}=0$ na equação (3). Vale salientar que é possível expressar a equação (7) em termos de sua função 
inversa, tal que

$$
I_{A}^{N P}\left(I_{E}^{N P}, d_{p}\right) \approx 1-J\left(\frac{J^{-1}\left(1-I_{E}^{N P}\right)}{\sqrt{d_{p}-1}}\right) .
$$

Curvas EXIT para um código LDPC de taxa $r_{c}=1 / 2$ e $E_{b} / N_{0}=1 \mathrm{~dB}$, para diversos valores de $d_{v}$ e $d_{p}$, são apresentadas em [3].

\section{Projeto de Códigos LDPC utilizando Curvas EXIT}

Um dos aspectos relevantes que é observado na análise de desempenho de esquemas de decodificação iterativa é a região da curva de probabilidade de erro de bit $\left(P_{b}\right)$ vs relação sinal-ruído $\left(E_{b} / N_{0}\right)$ em que ocorre uma queda abrupta da $P_{b}$ em função do número de iterações da decodificação. Essa região é denominada região de "queda d'água" (do inglês, water-fall) da curva $P_{b} \times E_{b} / N_{0}$ e é caracterizada pelo valor de $E_{b} / N_{0}$ que demarca o início da redução de $P_{b}$. Por comodidade, esse valor de relação sinal-ruído será denominado ponto-de-queda.

Por se tratar de códigos que usufruem das técnicas de decodificação iterativa, determinar o ponto-de-queda de códigos LDPC é uma importante tarefa no que se refere ao projeto de códigos. Para tanto, uma das formas usuais de se estimar o ponto-de-queda é por meio da geração das curvas $P_{b} \times E_{b} / N_{0}$ obtidas por simulações computacionais. Acontece que, dependendo do comprimento das palavras do código LDPC utilizado, as simulações podem se tornar bastante complexas devido à decodificação iterativa.

Para evitar tal desvantagem, as curvas EXIT podem ser utilizadas como uma ferramenta alternativa para a determinação do ponto-de-queda, que, neste caso, corresponde ao valor de $E_{b} / N_{0}$ para o qual as curvas EXIT dos decodificadores componentes se tocam. A Fig. 2 ilustra a obtenção do ponto-de-queda para um código LDPC $C(3,6)$ por meio da geração das curvas EXIT de seus decodificadores componentes.

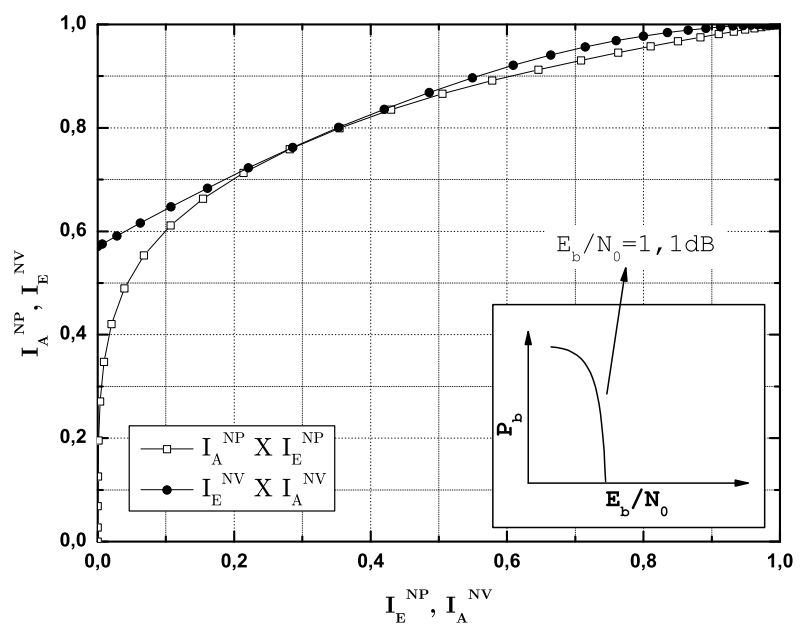

Fig. 2. Ponto-de-queda de um código LDPC obtido por meio de curvas EXIT.

Segundo a equação (3), a relação sinal-ruído tem influência direta no cálculo da informação mútua $I_{E}^{N V}$. Portanto, as curvas EXIT para o DNV variam de acordo com o valor de
$E_{b} / N_{0}$ adotado, conforme ilustra a Fig. 3, que também mostra a curva EXIT do DNP.

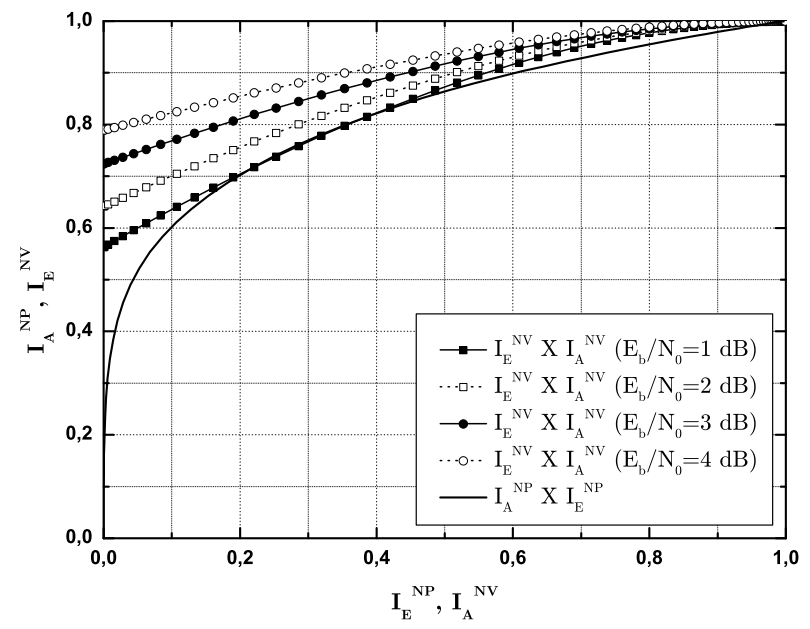

Fig. 3. Variação das curvas EXIT do DNV em função da relação sinal-ruído $E_{b} / N_{0}$.

Como a informação mútua $I_{E}^{N P}$ independe diretamente da relação sinal-ruído, fica fácil perceber que a curva EXIT do DNP não varia como a curva EXIT do DNV. Além disso, é importante ressaltar que a curva EXIT do DNP inicia sempre na origem dos eixos, já que no momento em que o receptor recebe o sinal proveniente do canal, os nós de verificação não possuem nenhuma informação a respeito do sinal recebido.

Um outro aspecto importante a ser considerado no projeto de um código LDPC é a sua complexidade de decodificação. A complexidade de um código LDPC pode ser relacionada à esparsidade de sua matriz de verificação de paridade $H$. A esparsidade da matriz $H$ está diretamente ligada aos pesos de suas colunas $\left(w_{c}\right)$ e de suas linhas $\left(w_{l}\right)$. Quanto menores os valores de $w_{c}$ e $w_{l}$, mais esparsa será a matriz $H$. Isto significa que quanto mais esparsa for a matriz $H$ de um código LDPC, menor será a sua complexidade de decodificação.

A complexidade de decodificação interfere diretamente no valor do ponto-de-queda de um código LDPC. Na Fig. 4 é indicada a variação dos valores de $E_{b} / N_{0}$ referentes aos pontos-de-queda de códigos LDPC regulares de taxa $r_{c}=1 / 2$ em função de $w_{c}$, sabendo-se que $w_{l}=2 w_{c}$. É possível observar que quanto mais esparsa for a matriz $H$, ou seja, quanto menor for a complexidade de decodificação do código LDPC, menor será o valor do respectivo ponto-de-queda.

As curvas EXIT ajudam a entender o comportamento da decodificação iterativa de códigos LDPC e indicam que existe um código de parâmetros $\left(w_{c}, w_{l}\right)$ e comprimento infinito, que atinge os limiares de decodificação obtidos. Para códigos de comprimento muito longos, a principal contribuição das curvas EXIT para o entendimento da decodificação iterativa é fornecer os limiares de convergência do algoritmo de decodificação apenas com a simulação dos decodificadores componentes, não havendo necessidade, portanto, da simulação completa do sistema.

\section{CURVAS EXIT PARA RECEPTORES ITERATIVOS}

Conceitos relevantes à aplicação de curvas EXIT no estudo de códigos LDPC foram expostos na Seção II. A partir deste 


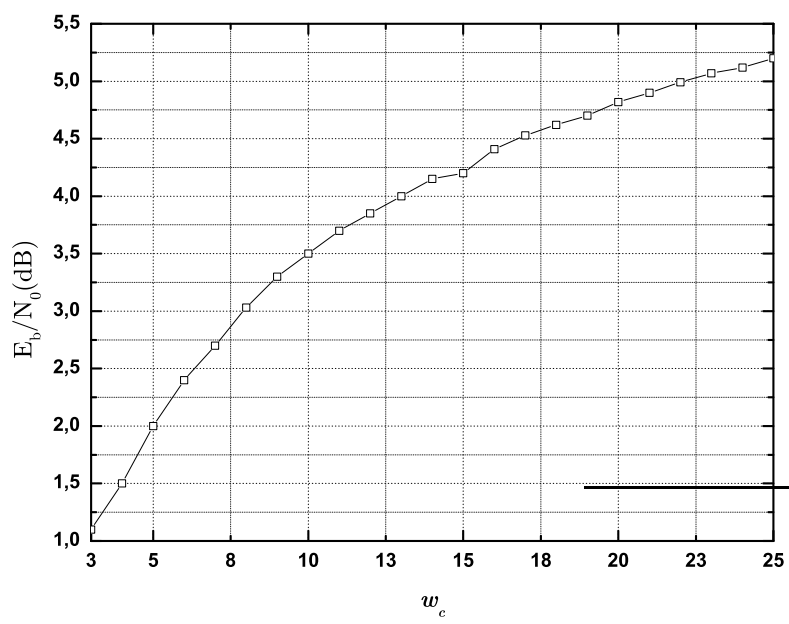

Fig. 4. Valores de ponto-de-queda para códigos LDPC de taxa $r_{c}=1 / 2 \mathrm{e}$ complexidades diferentes.

momento, iremos aplicar os conceitos definidos no sistema de comunicação apresentado em [5].

O transmissor é formado pela concatenação serial de um codificador LDPC e um modulador diferencial $M$-APSK (do inglês, M-ary Amplitude Phase Shift Keying). Neste esquema de modulação, os sinais são dispostos em $N$ anéis de amplitudes distintas, cada um contendo $P$ valores de fase alinhados. Os raios dos anéis diferem por um fator constante $r$ denominado razão de raio. Tais constelações são denotadas por $M-\operatorname{APSK}(N, P)$, com $M=N P$.

O modelo de canal considerado é o canal não-coerente de bloco, no qual a entrada é um vetor de comprimento $L, \mathbf{S}=$ $\left[s_{1}, s_{2}, \ldots, s_{L}\right]$, cujos elementos $s_{l}=a_{l} e^{j \phi_{l}}$ representam os símbolos APSK. A saída do canal também é um vetor de comprimento $L, \mathbf{R}=\left[r_{1}, r_{2}, \ldots, r_{L}\right]$, cujos elementos podem ser expressos como

$$
r_{l}=s_{l} \exp (j \theta)+n_{l}, l=1,2, \ldots, L
$$

em que $\theta$, a fase aleatória introduzida pelo canal, é representada por uma V.A. contínua com distribuição uniforme no intervalo $[0,2 \pi)$. A fase é considerada constante durante cada bloco de $L$ símbolos e varia de maneira independente de bloco a bloco. O ruído AWGN complexo é expresso por $n_{l}$, cujas componentes real e imaginária têm média zero e variância $\sigma_{n}^{2}=N_{0} / 2$ cada uma.

$\mathrm{O}$ projeto de sistemas que sejam robustos à variação do canal é um dos grandes desafios da comunidade científica. Para tentarmos nos aproximar do desempenho ótimo do canal Gaussiano com ruído de fase, podemos utilizar os receptores iterativos. Estes receptores são assim chamados por utilizarem algoritmos que executam conjuntamente estimação de canal, detecção e decodificação [11]. O grafo que representa o receptor iterativo abordado neste trabalho está ilustrado em [5].

Para que seja possível utilizar os conceitos apresentados na Seção II, o receptor iterativo será descrito pelo diagrama em blocos indicado na Fig. 5, na qual os parâmetros $B, D, F$ e $G$ representam as mensagens trocadas durante as operações de estimação do canal, demodulação e decodificação pelos blocos que compõem o receptor. Os blocos de nós de variável e de nós de verificação se referem aos decodificadores componentes do código LDPC, enquanto os blocos em cinza, denotados por Decodificador 1 e 2, representam os decodificadores aos quais as curvas EXIT se referirão deste ponto em diante. Para atingir este propósito, as mensagens empregadas no cálculo das informações mútuas serão as mensagens $D$ e $F$.

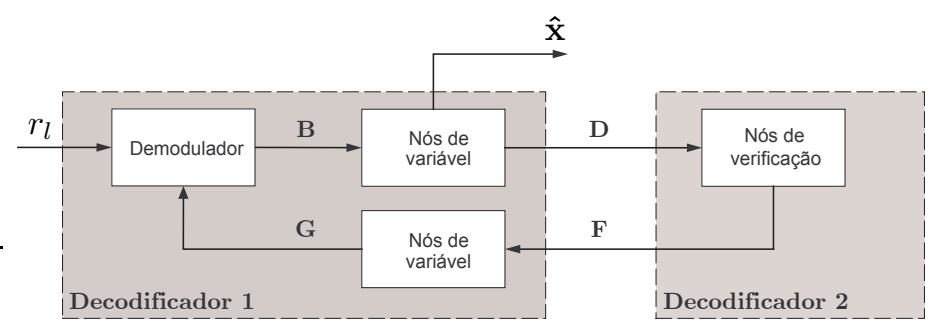

Fig. 5. Diagrama em blocos do receptor iterativo.

Antes de apresentarmos as curvas EXIT para o receptor iterativo em questão, é necessário que se entenda como ocorre a passagem de mensagens entre os blocos componentes indicados na Fig. 5. A explicação será dada a seguir:

1) Os símbolos recebidos $r_{l}$ chegam ao demodulador $\mathrm{e}$, juntamente com as mensagens $G$, dão origem às mensagens $B$, que são as mensagens de entrada do bloco de nós de variável;

2) No bloco de nós de variável, as mensagens $B$ são processadas e resultam nas mensagens de entrada do bloco dos nós de verificação (mensagens $D$ );

3) As mensagens $D$ são devidamente processadas no bloco de nós de verificação e, após serem convertidas nas mensagens $F$, são devolvidas ao bloco de nós de variável. A existência de dois blocos de nós de variável na Fig. 5 se deve ao fato de que as mensagens passadas por cada bloco são bem diferentes. No bloco inferior de nós de variável, as $d_{p}$ mensagens que vêm dos nós de verificação são somadas e entregues ao demodulador (mensagens $G$ ), enquanto no bloco superior as mensagens $B$ são calculadas segundo a equação (1), onde $Z_{i}$ é agora extraído de $\mathbf{R}$. Esta representação se justifica apenas para facilitar o entendimento.

Ainda sobre o diagrama em blocos que representa o receptor iterativo, é importante frisar que como o Decodificador 1 corresponde à concatenação do demodulador e o bloco de nós de variável, a equação (3) não pode ser mais utilizada para o cálculo da informação extrínseca, pois as mensagens que vinham antes da saída do canal (vide Fig. 1), agora vêm da saída do demodulador. Por outro lado, a equação (5) permanece sendo empregada para o cálculo das mensagens $F$, pois as mensagens $D$ continuam sendo as mensagens de saída dos nós de variável.

\section{A. Mensagens do Demodulador}

As mensagens de saída do demodulador, $B$, são calculadas a partir das mensagens provenientes do canal e das mensagens vindas dos nós de variável, $G$. As mensagens $G$ são computadas a partir da soma das mensagens oriundas dos nós de verificação ligados a cada nó de variável. Portanto, por meio 
de uma pequena modificação na equação (1), as mensagens $G$ podem ser obtidas pela expressão

$$
G=\sum_{j=1}^{d_{p}} F_{j} .
$$

Acontece que, na inicialização do algoritmo, as mensagens vindas do decodificador 2 correspondem a uma completa incerteza (zero no domínio dos valores- $L$ ). Logo, as mensagens $B$ são calculadas inicialmente com base apenas nas mensagens provenientes do canal.

Assumindo mais uma vez que as mensagens de saída do decodificador 2 são aproximadas por V.A.s Gaussianas, é possível utilizar novamente a função $J(\cdot)$ para a obtenção das curvas EXIT. Sendo assim, a informação mútua $I_{G}$ pode ser calculada por meio da equação [12]

$$
I_{G}=J\left(\sqrt{d_{v}} J^{-1}\left(I_{F}\right)\right)
$$

É importante salientar que dependendo da modulação empregada, as mensagens $B$ serão V.A.s cujas distribuições de probabilidade terão características diretamente relacionadas à modulação. Apesar de em [3] ter sido feita uma aproximação para as funções EXIT considerando tipos específicos de moduladores, nesse trabalho preferimos obter a distribuição de probabilidades na saída do demodulador por meio de simulação. Dessa forma, é possível fazer uma generalização para diferentes demoduladores.

As distribuições de probabilidades na saída do demodulador são calculadas considerando que as mensagens $G$ são obtidas a partir da soma de V.A.s Gaussianas. Face ao exposto, o desvio-padrão de $G$ é calculado por meio da função inversa de $J(\cdot)$, i.e., $\sigma_{G}=J^{-1}\left(I_{G}\right)$.

\section{B. Curvas EXIT para o Decodificador 1}

No Decodificador 1, as mensagens $G$ são enviadas ao demodulador para que a distribuição de probabilidades das mensagens $B$ seja obtida por meio de medidas de histograma. Feito isso, a informação mútua $I_{B}\left(\sigma_{G}\right)$ é calculada para cada valor de $\sigma_{G}$ da variável de entrada $G$, visto que $I_{G}$ é obtida por meio da equação (11).

Para o Decodificador $1, I_{F}$ representa a informação mútua a priori e $I_{D}$, a informação extrínseca. Logo, a informação mútua $I_{D}$ é calculada por

$$
\begin{aligned}
& I_{D}\left(I_{F}, d_{v}, \frac{E_{b}}{N_{0}}, r_{c}\right)= \\
& \quad J\left(\sqrt{\left(d_{v}-1\right)\left[J^{-1}\left(I_{F}\right)\right]^{2}+\left[J^{-1}\left(I_{B}\right)\right]^{2}}\right) .
\end{aligned}
$$

Note que a equação (12) é obtida substituindo-se $\sigma_{Z}$ por $J^{-1}\left(I_{B}\right)$ na equação (3), semelhante ao que foi feito em [12]. A função EXIT do Decodificador 1 é aproximada pela função $J(\cdot)$ pelo fato de as mensagens $D$ serem as mensagens de saída dos nós de variável. Os valores de $I_{B}$ são calculados através dos resultados de simulação computacional do demodulador.

\section{Curvas EXIT para o Decodificador 2}

Uma vez que o demodulador está conectado aos nós de variável, é possível afirmar que apenas a curva EXIT do Decodificador 1 será influenciada pela presença do demodulador. Dessa forma, as operações realizadas pelo decodificador 2 são determinadas pela equação (5), indicando que o Decodificador 2 se assemelha ao DNV indicado na Fig. II. A curva EXIT do Decodificador 2 pode ser obtida por meio das equações (7) e (14), reescritas como

$$
I_{F}\left(I_{D}, d_{p}\right) \approx 1-J\left(\sqrt{d_{p}-1} \cdot J^{-1}\left(1-I_{D}\right)\right)
$$

e

$$
I_{D}\left(I_{F}, d_{p}\right) \approx 1-J\left(\frac{J^{-1}\left(1-I_{F}\right)}{\sqrt{d_{p}-1}}\right) .
$$

em que $I_{D}$ é a informação mútua intrínseca e $I_{F}$, a informação mútua extrínseca do Decodificador 2.

\section{RESULTADOS}

Na Seção II, foi apresentado o procedimento de análise da decodificação iterativa de códigos LDPC por meio de curvas EXIT. Tal procedimento foi modificado e estendido para avaliar a demodulação e decodificação conjuntas do receptor iterativo, apresentado na Seção III de [5]. Para facilitar o entendimento, as informações mútuas definidas nas Subseções III-B e III-C, quais sejam $I_{D}$ e $I_{F}$, serão denotadas por $I_{E}^{D E C 1}$ $\left(I_{A}^{D E C 2}\right)$ e $I_{A}^{D E C 1}\left(I_{E}^{D E C 2}\right)$, quando o Decodificador 1 (2) for considerado.

A partir daqui, apresentaremos os resultados obtidos com a aplicação do procedimento em questão no receptor iterativo de um sistema de comunicação que considera o transmissor e o canal descritos na Seção III. Foram utilizados um código LDPC regular $C(3,6)$ de taxa $r_{c}=1 / 2$ e um esquema de modulação 8-APSK, com razão de raio $r=2,42$, assim como em [5]. Os resultados obtidos foram baseados na variação do intervalo de coerência do canal, ou seja, no parâmetro $L$. Desta forma, foi possível fazer uma previsão do comportamento da decodificação iterativa em função do tamanho do bloco de memória do canal. Resultados referentes ao estudo da capacidade de canais $M$-APSK/AWGN não coerentes de bloco apresentados em [6] possibilitaram a estimação dos valores de relação sinal-ruído, $R S R_{L}$, para a capacidade do canal 8-APSK/AWGN com $L=9\left(R S R_{9}=1,7 \mathrm{~dB}\right)$ e $L=29$ $\left(R S R_{29}=1,4 \mathrm{~dB}\right)$, indicados em [5]. Estes valores de relação sinal-ruído foram utilizados como meta de desempenho para o receptor iterativo apresentado.

Conforme mencionado anteriormente, a simulação computacional é uma das formas de se obter o ponto-de-queda do sistema proposto. Entretanto, esta técnica pode se mostrar um tanto ineficiente à medida que o comprimento do código aumenta. Em outras palavras, o aumento do comprimento do código torna maior a latência do decodificador e, consequentemente, deixa a decodificação iterativa mais complexa. Sendo assim, a utilização de curvas EXIT para estimar o ponto-de-queda simplifica a investigação do receptor iterativo em questão.

As curvas EXIT dos Decodificadores 1 e 2, considerando um canal 8-APSK/AWGN com $L=9, L=20$ e $L=$ 
29 , estão ilustradas nas Figs. 6, 7 e 8 , respectivamente. Para a geração dos valores das mensagens $B$ na saída do demodulador, foi utilizado o mesmo cronograma de passagem de mensagens adotado em [5], com pequenas modificações nos valores iniciais de algumas mensagens.

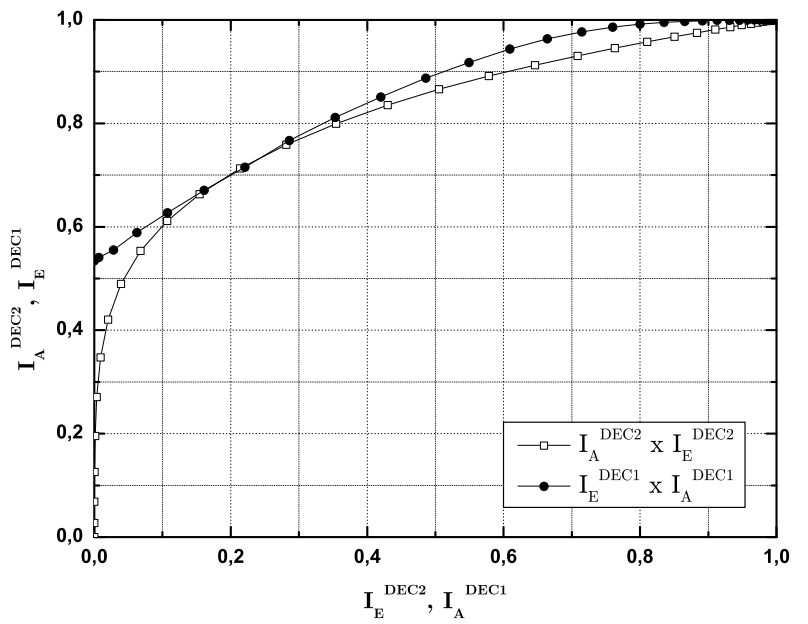

Fig. 6. Curvas EXIT obtidas para o canal 8-APSK/AWGN com $L=9$ e código LDPC $C(3,6)$.

O cálculo das curvas EXIT permitiu a obtenção dos pontos-de-queda do receptor iterativo estudado. Os valores obtidos foram $4,7 \mathrm{~dB}(L=9), 2,6 \mathrm{~dB}(L=20) \mathrm{e}$ $2,5 \mathrm{~dB}(L=29)$, indicando que quanto maior o valor de $L$, menor o ponto-de-queda. Todavia, a diminuição do valor do ponto-de-queda não ocorre de maneira linear, quando comparada ao aumento do intervalo de coerência do canal.

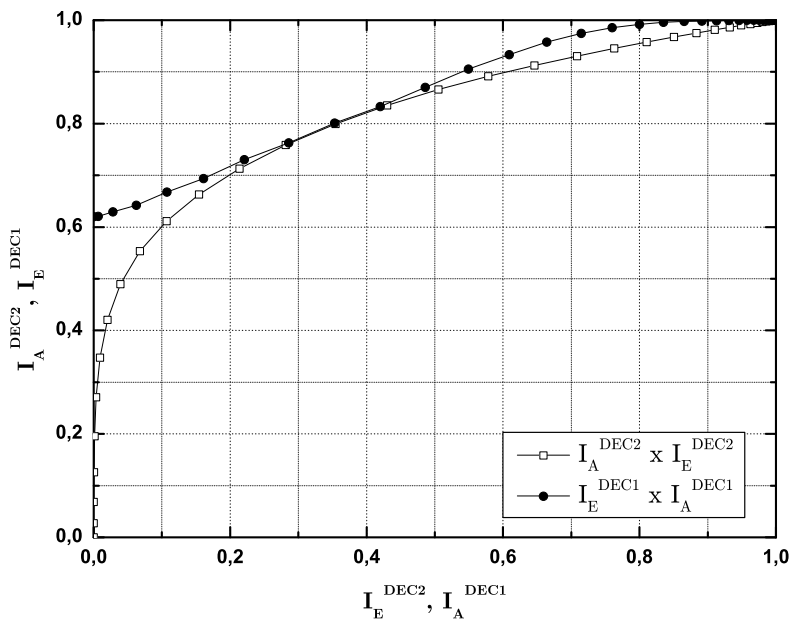

Fig. 7. Curvas EXIT obtidas para o canal 8-APSK/AWGN com $L=20$ e código LDPC $C(3,6)$.

\section{COMENTÁRIOS FINAIS}

É importante destacar que os casos abordados neste trabalho consideraram apenas a utilização de códigos LDPC regulares. Ficou constatado que o ponto-de-queda do receptor iterativo ainda está distante da capacidade de canal para pequenos valores do intervalo de coerência do canal $M$-APSK/AWGN (3 $\mathrm{dB}$ para $L=9$ ). Entretanto, para valores médios do intervalo de coerência, o ponto-de-queda se aproxima da capacidade $(1,1 \mathrm{~dB}$ para $L=29)$. Também podemos concluir que a previsão do limiar de desempenho do receptor

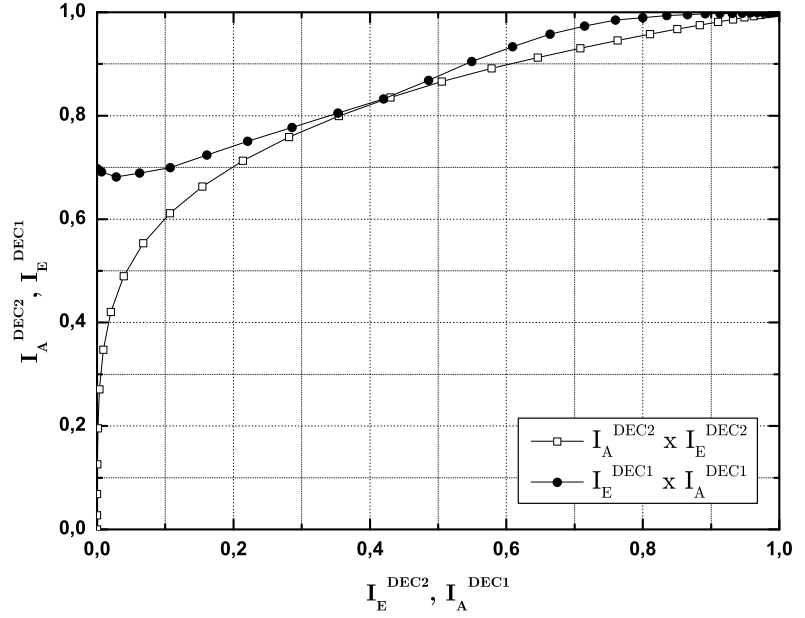

Fig. 8. Curvas EXIT obtidas para o canal 8-APSK/AWGN com $L=29$ e código LDPC $C(3,6)$.

iterativo proposto para o canal com memória limitada pode ser desenvolvida através do uso de curvas EXIT. Em particular, as funções EXIT nos indicam que existe um código LDPC regular que pode levar o ponto-de-queda a valores relativamente próximos da capacidade de canal para um intervalo de coerência suficientemente grande. Como proposta futura de trabalho, pretendemos determinar qual o comprimento finito de código LDPC que se aproxima da previsão das curvas EXIT.

\section{REFERÊNCIAS}

[1] S. ten Brink, "Convergence of Iterative Decoding," Electronics Letters, vol. 35, n. 13, pp. 806-808, Jun 1999.

[2] S. ten Brink, "Convergence Behavior of Iteratively Decoded Parallel Concatenated Codes," IEEE Trans. on Communications, vol. 49, n. 10 , pp. 1727-1737, Out 2001.

[3] S. ten Brink, G. Kramer e A. Ashikhmin "Design of Low-Density Parity-Check Codes for Modulation and Detection," IEEE Trans. on Communications, vol. 52, n. 4, pp. 670-678, Abr 2004.

[4] A. Ashikhmin, G. Kramer e S. ten Brink, "Extrinsic Information Transfer Functions: A Model and Two Properties," IEEE Trans. on Information Theory, vol. 50, n. 11, pp. 2657-2673, Nov 2004.

[5] D. C. Cunha e J. Portugheis, "Blockwise Noncoherent $M$-APSK Channel: Coding Scheme for Iterative Receiver," Em Anais do XXV Simpósio Brasileiro de Telecomunicações (SBrT 2007), Recife-PE, Set 2007.

[6] D. C. Cunha e J. Portugheis, "Capacity of Blockwise Noncoherent $M$-APSK/AWGN Channel," Electronics Letters, vol. 42, n. 14, pp. 816-817, Jul 2006.

[7] F. R. Kschischang, B. J. Frey e H. -A. Loeliger, "Factor Graphs and the Sum-Product Algorithm," IEEE Trans. on Information Theory, vol. 47, n. 2, pp. 498-519, Fev 2001.

[8] J. Hagenauer, E. Offer e L. Papke, "Iterative Decoding of Binary Block and Convolutional Codes," IEEE Trans. on Information Theory, vol. 42, n. 2, pp. 429-445, Mar 1996.

[9] M. Ardakani e F. R. Kschischang, "A More Accurate One-Dimensional Analysis and Design of Irregular LDPC Codes," IEEE Trans. on Communications, vol. 52, n. 12, pp. 2106-2114, Dez 2004.

[10] S. Y. Chung, R. Urbanke e T. J. Richardson, "Analysis of Sum-Product Decoding of Low-Density Parity-Check Codes Using a Gaussian Approximation," IEEE Trans. on Information Theory, vol. 47, n. 2, pp. 657-670, Fev 2001.

[11] A. P. Worthen e W. E. Stark, "Unified Design of Iterative Receivers using Factor Graphs," IEEE Trans. on Information Theory, vol. 47, n.2, pp. 843-849, Fev 2001.

[12] M. Franceschini, G. Ferrari, R. Raheli e A. Curtoni, "Serial Concatenation of LDPC Codes and Differential Modulations," IEEE Journal on Selected Areas in Communications, vol.23, n.9, pp. 1758-1768, Set 2005. 